Meta

Journal des traducteurs

Translators' Journal

\title{
La place de l'interprétation dans l'itinéraire scolaire, professionnel et universitaire d'un sourd
}

\section{Olivier Delanghe}

Volume 42, numéro 3, septembre 1997

L'interprétation en langues des signes

URI : https://id.erudit.org/iderudit/002505ar

DOI : https://doi.org/10.7202/002505ar

Aller au sommaire du numéro

Éditeur(s)

Les Presses de l'Université de Montréal

ISSN

0026-0452 (imprimé)

1492-1421 (numérique)

Découvrir la revue

Citer cet article

Delanghe, O. (1997). La place de l'interprétation dans l'itinéraire scolaire, professionnel et universitaire d'un sourd. Meta, 42(3), 555-559.

https://doi.org/10.7202/002505ar
Résumé de l'article

Olivier Delanghe, sourd profond depuis l'âge de un an, relate son itinéraire. Tout d'abord sa scolarité : l'enseignement qu'il reçoit est peu adapté à son état de sourd profond et ne lui permet pas d'obtenir le baccalauréat. Il se découvre, à travers ses difficultés, une vocation d'enseignant. Le professorat ne lui étant pas accessible, il suit une formation d'éducateur spécialisé. La présence d'un interprète en LSF (langue des signes française) lui ayant permis de comprendre les cours à égalité avec les entendants, il obtient son diplôme. Il assure aujourd'hui un cours de perfectionnement de la LSF dans le cadre de la formation d'interprètes mise en place à l'ESIT. 


\title{
LA PLACE DE L'INTERPRÉTATION DANS L'ITINÉRAIRE SCOLAIRE, PROFESSIONNEL ET UNIVERSITAIRE D'UN SOURD
}

OLIVIER DELANGHE

Université Paris III-Sorbonne Nouvelle (ESIT), Paris, France

\begin{abstract}
Résumé
Olivier Delanghe, sourd profond depuis l' âge de un an, relate son itinéraire. Tout d'abord sa scolarité : l'enseignement qu' il reçoit est peu adapté à son état de sourd profond et ne lui permet pas d'obtenir le baccalauréat. Il se découvre, à travers ses difficultés, une vocation d'enseignant. Le professorat ne lui étant pas accessible, il suit une formation d'éducateur spécialisé. La présence d' un interprète en LSF (langue des signes française) lui ayant permis de comprendre les cours à égalité avec les entendants, il obtient son diplôme.

Il assure aujourd' hui un cours de perfectionnement de la LSF dans le cadre de la formation d'interprètes mise en place à l'ESIT.

Abstract

Olivier Delanghe has been deaf since he was one year old. He attended primary and secondary school but, as there was insufficient provision made for deaf students he was unable to graduate.

The difficulties he experienced led him to embrace the teaching profession. He entered a specialized trainig course providing sign language interpreting and successfully graduated. At present he teaches LSF at ESIT.

His failures and successes show how important it is for deaf people to have a good command of sign language, and the vital role played by interpreters in improving the status of the Deaf.
\end{abstract}

Mes parents sont entendants. Je suis sourd profond. Je suis devenu sourd à l'âge de un an. Dès mon plus jeune âge, j'ai été scolarisé dans une école spécialisée dans la prise en charge des sourds à Lille. La pédagogie était oraliste, les enseignants ne connaissaient pas la langue des signes. Par contre, les élèves signaient ${ }^{1}$ entre eux. Vers six ou sept ans, nous étions plus à même de nous exprimer en LSF qu'en français. En fait, nous signions et en même temps nous labialisions ${ }^{2}$ des mots français. Par exemple, nous avions l'habitude d'associer au signe manuel unique signifiant jamais vu l'articulation jamais vu, idem pour même temps. Le professeur ne nous avait pas enseigné ces mots, nous ne pouvions pas lui demander la moindre explication à ce propos, il ne connaissait pas la LSF. J'avais appris, comme tous mes camarades, la LSF auprès d'élèves plus anciens en arrivant à l'école. Ce n'est que vers dix ou onze ans, à l'occasion d'une leçon de français, où j'ai dû déchiffrer jamais $v u$ écrit au tableau, que je me suis aperçu qu'il ne s'agissait pas d'un mot unique comme je me l'imaginais mais de deux mots. Du même coup, j'ai fait le lien entre ces mots écrits du français et ce que $\mathrm{j}$ 'articulais. Un ami sourd $\mathrm{m}$ 'a dit qu'il avait vécu la même expérience. Avec le recul, je me dis que nous avons perdu beaucoup de temps, je savais dire ces mots et $j$ 'en connaissais parfaitement la signification quatre ans avant qu'ils ne me soient présentés par le professeur.

Meta, XLII, 3, 1997 
À treize ans ${ }^{3}$, je suis allé au collège spécialisé à Arras où les enseignants utilisaient la même méthode qu'à Lille. Ils ignoraient la LSF, mais il faut reconnaître qu'ils avaient une très bonne articulation, ce qui facilitait la lecture labiale. De manière générale, $j$ 'avais de bons résultats pour tous les exercices de conjugaison, de grammaire, de logique et de mathématiques. Mes résultats étaient plus médiocres en rédaction. J'ai eu réellement envie de lire lorsque je suis entré en classe de quatrième, $\mathrm{j}$ 'ai compris à cette époque l'importance du français, de la lecture, de l'écriture. C'est à partir de ce moment-là que j'ai commencé à progresser en français.

Arrivé en troisième à 17 ans, j'ai changé d'établissement. Je suis allé à Nevers dans une classe intégrée ${ }^{4}$ où tous les élèves étaient malentendants, j'étais le seul sourd profond. Les élèves malentendants ne connaissaient pas la LSF. Contrairement aux enseignants auxquels j'avais eu affaire jusqu'à présent, les professeurs à Nevers n'étaient pas spécialisés dans la prise en charge des sourds. Il s'agissait d'enseignants ordinaires qui accueillaient des malentendants. Au fil des ans, ils étaient devenus assez au fait de leurs problèmes, mais ils n'étaient pas en mesure de s'adapter à un sourd profond comme moi.

Dès que je me suis retrouvé dans cette classe, le français m'est apparu comme très important. Ce n'était pas seulement une matière en soi, mais aussi le support de toutes les autres matières au travers des énoncés des problèmes de mathématiques ou de physique, des définitions, des textes des manuels, etc. Dès que j'ai été confronté à tous ces types d'écrit, je me suis rendu compte que le français qu'on m'avait proposé dans les établissements spécialisés avait été très simplifié. J'ai eu la chance de trouver une orthophoniste tout à fait compétente. À raison de trois séances hebdomadaires, et ce, pendant quatre ans, elle m'a aidé à combler mon retard. Pour être tout à fait exact, il faut dire que mes capacités de compréhension du français ont progressé plus vite que mes capacités d'expression. Toujours est-il qu'au bout de ces quatre années, il m'a été possible de lire à peu près n'importe quel texte.

Malgré tous mes efforts, j'ai échoué au baccalauréat. L'enseignement que j'avais reçu n'avait pas été adapté au sourd profond que je suis. Pendant les cours, l'information ne me parvenait jamais dans son intégralité. Lorsque le professeur posait oralement une question, je n'en saisissais que des bribes. Je ne pouvais pas y répondre. La communication n'était jamais ni fluide ni aisée. L'image qui me vient à l'esprit pour décrire ce que je ressentais est celle d'un cycliste dont le pédalier ne fonctionnerait que par à-coups.

Après cet échec, j'en étais venu à me dire que mes professeurs avaient raison de penser que je n'étais pas capable. Je doutais de moi. Pendant les quatre années passées à Nevers et aussi à ce moment-là, mon orthophoniste m'avait toujours encouragé et soutenu. Je me souviens d'une conversation avec elle. Je lui avais demandé quel métier m'aurait-elle bien vu exercer. Elle m'avait répondu : «Professeur!» C'était justement ce à quoi je rêvais secrètement : enseigner aux enfants sourds, les aider. Elle avait, m'expliqua-t-elle, repéré chez moi des qualités de pédagogue. Au cours des nombreuses séances de travail, elle me demandait souvent, une fois que $\mathrm{j}$ 'avais acquis telle ou telle notion, comment l'expliquer à des sourds. Mes réponses avaient dû lui paraître appropriées.

C'est dans l'établissement où j'avais démarré ma scolarité, à Lille, que j'ai trouvé mon premier emploi. Cet établissement, tout en restant à Lille, avait déménagé et, à mon étonnement, il avait dans le même temps changé d'orientation. En outre, il accueillait désormais des sourds plurihandicapés pour qui la méthode oraliste n'était pas pertinente. J'intervenais donc en LSF auprès de ces enfants. Le directeur de cet établissement me proposa de suivre une formation d'éducateur. Au départ, $\mathrm{j}$ 'avais des réticences, $\mathrm{j}$ 'imaginais que la psychologie occupait une part très importante dans la formation et je n'avais a priori pas d'attirance pour cette discipline. Malgré cela, j'ai accepté de suivre un stage 
d'une semaine à l'école d'éducateurs spécialisés de Montpellier. Pendant cette semaine d'évaluation, nous eûmes beaucoup d'échanges; on nous présenta in extenso le programme de formation. Mes réticences s'évanouirent lorsque je réussis à faire un lien entre les enseignements qui nous étaient proposés et ma pratique auprès des enfants. En outre, un élément déterminant me décida: les cours seraient interprétés en LSF. Il faut préciser que cette formation d'éducateur spécialisé, ouverte aux sourds, se déroulait dans une école d'éducateurs ordinaire. La promotion à laquelle j'ai été intégré comprenait quarante étudiants, six sourds et trente-quatre entendants.

Pour la première fois de ma vie, j'ai suivi les cours par le truchement d'un interprète. Quel soulagement! Je n'avais plus de problèmes. Bien sûr, je connaissais l'existence des interprètes, $j$ 'en avais vus à la télévision, $j$ 'avais suivi des conférences par leur intermédiaire, mais c'est à l'occasion de cette formation que j'ai pu apprécier pleinement le bénéfice que je pouvais tirer de la présence d'un interprète.

Pour la première fois de ma vie, $\mathrm{j}$ 'avais accès au contenu comme les autres sans devoir fournir un effort harassant. Dans le même temps, j'ai éprouvé un regain d'intérêt pour la langue, pour les langues.

Avec l'interprète, nous avons dû créer un code signé pour les notions inédites en LSF. Cela m'a beaucoup intéressé. Nous parlions de code et non pas de néologisme, car nous étions en butte aux critiques d'une fraction de la communauté sourde qui craignait que ces créations portent atteinte à la pureté de la LSF. Néologisme, code, peu importe le vocable, toujours est-il que cette activité de création de signe nous obligeait à approfondir le contenu des notions. Qui plus est, je me suis aperçu que lorsque j'abordais des textes traitant des sujets que nous avions approfondis, ma lecture s'en trouvait grandement facilitée.

Pendant les cours, l'interprète alternait deux modes de traduction. Il restituait en français signé ${ }^{5}$ certaines tournures ou expressions françaises, propres à la matière enseignée. Il procédait ainsi à notre demande, car nous souhaitions pouvoir les utiliser par la suite à l'écrit. L'interprétation nous permettait d'enrichir notre français. Un ami sourd, qui est allé étudier aux États-Unis, m'a dit que là-bas les étudiants avaient les mêmes exigences s'agissant de l'anglais. L'interprète n'avait recours au français signé que de façon ponctuelle, lorsqu'il sentait que telle ou telle tournure française devait nous être restituée telle quelle. Pour le reste, il interprétait idiomatiquement en LSF.

Grâce à l'interprétation, je me rendis compte que certains professeurs étaient ennuyeux, d'autres très vivants. Je fis aussi d'autres découvertes.

À l'occasion de travaux de groupe, les sourds étaient répartis dans ces groupes, j'ai eu l'occasion de me confronter aux entendants. Je me suis aperçu que je pouvais comprendre, le cas échéant, mieux que certains entendants. Auparavant, je pensais, n'ayant jamais été intégré dans une formation ordinaire, que les entendants comprenaient tout parce qu'ils entendaient et aussi parce que depuis mon enfance j'avais toujours été obligé de demander des explications aux entendants. Quand, par hasard, l'un d'entre eux me disait «je ne comprends pas, je ne sais pas», je trouvais cela bizarre, j'avais du mal à l'admettre. Depuis, $j$ 'ai compris qu'en la matière le fait d'être entendant ne constitue pas un viatique. En outre, dans ces groupes, nous devions rédiger collectivement. Les entendants proposaient parfois des formulations qui me paraissaient compliquées et alambiquées. $J$ 'avais quelques réticences à proposer les énoncés que j'avais préparés, j'avais peur qu'on les trouve trop simples. J'ai été très surpris lorsque, de temps en temps, ils ont été préférés à d'autres parce que plus clairs. À l'occasion de ces travaux de groupe, j'ai pu aussi m'apercevoir que chacun avait une personnalité propre à l'écrit et que ce n'était pas seulement le fait des écrivains. La confrontation à autrui, dans le cadre de cette formation, m'a aidé à évoluer sur plusieurs points. Je n'ai plus pensé la confrontation entre sourds et entendants en termes 
de supériorité ou d'infériorité. Auparavant, j'étais très tranché dans mes prises de position sans aller vraiment au fond des choses; peu à peu, j'ai appris à prendre du recul, à nuancer et à approfondir mes jugements.

L'école de Montpellier a fait preuve de souplesse dans l'accueil des sourds. Nous étions soumis au même régime que les entendants pour l'évaluation. La seule différence portait sur les travaux écrits, les enseignants avaient pris en compte les difficultés des sourds en français et étaient un peu moins exigeants en la matière. Il va de soi que cette indulgence ne valait que pour des maladresses au plan formel et qu'en ce qui concerne les contenus, nous étions logés à la même enseigne que les entendants. Le bénéfice que j'ai retiré de cette formation, outre le fait que j'ai obtenu mon diplôme d'éducateur spécialisé au bout de deux ans, c'est que j'ai retrouvé toute la confiance en moi que j'avais perdue lors de mon séjour au lycée.

L'interprète a joué un rôle important dans cette réussite. J'ai oublié de dire qu'au début de la formation, il a eu aussi en quelque sorte un rôle de médiateur entre les sourds et les entendants qui se retrouvaient réunis pour la première fois. À dire vrai, son rôle de médiateur a été essentiel mais bref. Rapidement, la présence de sourds dans l'établissement est devenue quelque chose de très banal.

À l'heure actuelle, je suis enseignant à ''INJS $^{6}$ de Paris dans le cadre d'une filière bilingue $^{7}$. Parallèlement, je prépare un DEUG de linguistique. Mes enseignants sont compréhensifs. Je n'ai d'interprète ni pour les cours ni pour passer les examens, aucune prise en charge au plan financier n'a pu être trouvée. Pendant les cours, je prend des notes en copiant sur un voisin. Malheureusement, les notes que prennent les étudiants ne sont pas complètes et souvent très personnelles. Les professeurs me donnent un résumé des cours. Je dois m'accrocher, ce n'est pas facile. Je ne pense pas que j'aurais pu poursuivre ces études d'emblée dans de telles conditions. L'expérience que j'ai vécue à Montpellier, pour toutes les raisons que j'ai dites précédemment, m'y a largement préparé.

Enfin, depuis janvier 1994, j'assure un cours de perfectionnement linguistique en LSF pour interprètes dans le cadre de la formation donnée à l'ESIT. Lorsque j'ai accepté de prendre en charge ce cours, je me suis posé des questions. Que pouvais-je apporter à des entendants qui connaissaient déjà la LSF? J'avais peur d'aborder des aspects de la langue qu'ils maîtrisaient déjà. Il est un peu tôt pour dresser un bilan. Ce que je peux indiquer c'est que, après une période d'ajustement et de mise en place, l'essentiel de ces cours a consisté en un travail sur la prise en charge en LSF de discours ne relevant pas d'échanges courants et tout particulièrement sur l'introduction de concepts inédits en LSF. Cet aspect de l'interprétation en LSF est capital. En effet, c'est bien là tout le but de la formation d'interprètes de haut niveau destinés à faciliter l'insertion des sourds à un niveau plus élevé dans la société. Comme je l'ai indiqué précédemment, introduction de concepts ne signifie pas création de néologismes. Cette création relève d'un travail concerté auquel les sourds doivent prendre pleinement part. Cela dit, l'interprétation de conférence est en soi un exercice difficile; la nécessité d'introduire des concepts inédits en langue des signes complique la tâche des interprètes, d'où l'importance de la formation mise en place à l'ESIT.

J'aimerais ajouter qu'au fur et à mesure que les sourds ont accès aux domaines les plus variés, la linguistique, les sciences de l'éducation ou la psychologie, par exemple, la langue des signes doit s'enrichir de nouveaux concepts. S'agissant de la linguistique, des concepts tels que variante, diglossie, norme, pidgin n'ont pas encore de signe. Ce n'est pas tout, la langue des signes doit aussi s'enrichir au fur et à mesure que son utilisation se développe dans le champ scolaire, et ce, quel que soit le niveau considéré. Dans le cadre de mon activité d'enseignant auprès de jeunes sourds, j'ai pour ma part introduit des signes pour des concepts inédits en LSF, pour élément, par exemple. J'introduis le signe après explication du mot et l'utilise couramment dans la classe. Il est réutilisé par les 
élèves, et ce, dans divers contextes. L'introduction du signe renforce chez les élèves l'assimilation du concept. Cela vaut aussi, bien sûr, pour les adultes et les étudiants sourds. On l'aura compris, il ne s'agit surtout pas de mettre en concurrence la langue des signes et le français. Bien au contraire, plus la langue des signes sera riche, meilleur sera l'accès des sourds au français.

\section{Notes}

1. Signer, c'est-à-dire s'exprimer en langue des signes.

2. Labialiser, articuler sans voix de manière à produire un signifiant visuel lu sur les lèvres.

3. L'âge normal d'entrée au collège est de onze ans. Ce retard de deux ans est tout à fait dans l'ordre des choses s'agissant d'un élève sourd.

4. Classe de déficients auditifs installée dans des établissements ordinaires de l'Éducation Nationale.

5. Transcodage signé à partir du français.

6. Institut National de Jeunes Sourds.

7. Le projet pédagogique associe la LSF au français parlé et écrit. 